\title{
Caracterización morfológica de los recubrimientos 140MXC-530AS y 140MXC-560AS usando la técnica de proyección térmica por arco eléctrico
}

\author{
Morphological Characterization of Coatings 140MXC-530AS and \\ 140MXC-560AS Using the Electric Wire Arc-Spraying Process
}

\author{
Rojas-Molano Héctor Fernando \\ Universidad Libre de Colombia \\ Facultad de Ingeniería \\ Correo:hefrojasmo@unal.edu.co \\ Olaya-Flórez Jhon Jairo \\ Universidad Nacional de Colombia \\ Correo:jjolaya@unal.edu.co
}

Molina-González Cesar Andrés

Universidad Libre de Colombia

Información del artículo: recibido: junio de 2014, reevaluado: agosto de 2014, aceptado: junio de 2015

\begin{abstract}
Resumen
En este estudio se produjeron los recubrimientos 140MXC-530AS y 140MXC560AS, cuyas características corresponden a materiales nanoestructurados (140MXC) y materiales convencionales como aceros al carbono (530AS) e inoxidables (560AS), aplicados sobre un sustrato AISI-SAE 4340, por medio de la técnica de aspersión térmica por arco eléctrico, teniendo en cuenta los parámetros de depósito tales como corriente $(\mathrm{I})$, voltaje $(\mathrm{V})$, presión de aire primaria (AP) y presión de aire secundaria (AS), empleando un diseño Taguchi fraccional-factorial $\mathrm{L}_{9}\left(3^{4-2}\right)$. La caracterización de los depósitos se realizó mediante las técnicas de fluorescencia de Rayos X (XRF), microscopia óptica convencional (COM), microscopia electrónica de barrido (SEM), microscopía de fuerza atómica (AFM) y microdureza. Los resultados de esta caracterización se analizaron en función del espesor, microdureza, tamaño de partículas semifundidas y rugosidad del recubrimiento obtenido.
\end{abstract}

\section{Descriptores:}

- aspersión térmica

- caracterización

- diseño Taguchi

- recubrimientos 


\begin{abstract}
In this study were produced the 140MXC-530AS and 560AS 140MXC-coatings, whose characteristics correspond to nanostructured materials (140MXC) and conventional materials such as carbon steel (530AS) and stainless steel (560AS). This coatings were applied on a substrate of AISI-SAE 4340, through the technique of electric wire arc spraying, considering the deposition parameters such as current (I), voltage $(V)$, primary air pressure $(A P)$ and secondary pressure air (AS) using a fractional-factorial design $L_{g}\left(3^{4-2}\right)$ of Taguchi. The characterization of the mixtures were performed by the techniques of X-ray fluorescence (XRF), conventional optical microscopy (COM), scanning electron microscopy (SEM), atomic force microscopy (AFM) and microhardness. These results was analyzed in terms of the thickness, microhardness, and particle size of semi-molten coating obtained roughness.
\end{abstract}

\section{Introducción}

El proceso de proyección térmica es la aportación de materiales depositados en forma de partículas fundidas finamente divididas sobre un substrato debidamente preparado; este proceso se puede agrupar en tres grandes categorías: proyección a la llama, por arco eléctrico y plasma; mientras que los recubrimientos se pueden aplicar en forma de polvos, alambres o varillas, que cuando impactan sobre la superficie generan una acumulación de las partículas creando una estructura o película laminar delgada (Handbook of Thermal Spray Technology, 2004).

En la técnica de proyección térmica por arco eléctrico, se alimentan dos alambres a la pistola de proyección que sirven como electrodos; estos se encuentran inicialmente aislados uno del otro y avanzan de manera simultánea y automática, movidos por el equipo para encontrarse en un punto por delante de la fuente de gas que transportará las partículas fundidas generadas por un corto circuito al entrar en contacto. El chorro de gas comprimido fluye a través de la zona del arco y proyecta el metal líquido en forma de gotas fundidas sobre el substrato (Kelly, 2003).

Esta técnica proporciona recubrimientos de protección contra desgastes abrasivos y corrosión; pero para lograrlo satisfactoriamente se hace necesario controlar mejor la técnica al momento de preparar y aplicar el recubrimiento (Thermal Spray Society Training Committee, 2004). Los parámetros de depósito que se utilizan principalmente son: la corriente, el voltaje y las presiones de aire primario y secundario; todos ellos muchas veces no tienen la misma contribución sobre los efectos en las propiedades del recubrimiento obtenido (Cooke et al., 2007).

El recubrimiento a obtenerse es una región superficial de un material con propiedades diferentes de las del material base (Salvador, 2001). Su propósito es reemplazar, modificar y lubricar superficies. Además optimiza la superficie del material y le da resistencia mecánica al desgaste y a la fricción, además de ligereza y permite el aislamiento térmico y eléctrico (Quaranta et al., 2004). Las características para los distintos procesos de recubrimientos, dependen de las temperaturas de trabajo y de los espesores obtenidos (Knight, 1998). Para esta investigación se utilizó el alambre 140MXC con estructura nanocristalina y amorfas, caracterizado por su baja porosidad, alta dureza, resistencia al desgaste y a la corrosión y distribución homogénea de las partículas (Praxair TAFA 1.9.2). Adicionalmente se emplearon el 530AS con composición similar a un acero de medio contenido de carbono, apropiado para una amplia gama de aplicaciones donde la dureza no es relevante, los espesores requeridos no son demasiado grandes y su bajo costo es vital (Castolin Eutectic, Arc 530 Wire) y el 560AS con composición similar a la de un acero inoxidable 420, con buena resistencia a la abrasión y corrosión, ampliamente utilizado para la reparación dimensional de máquinas y aplicaciones de restauración de elementos tridimensionales, excelente para aplicaciones que requieren una capa de espesores considerables de recubrimiento (Castolin Eutectic, Arc 560 Wire).

El diseño experimental Taguchi se utilizó para originar los recubrimientos 140MXC-530AS y 140MXC560AS, que se depositaron sobre el sustrato AISI-SAE 4340 y cuya composición química es ideal para dispositivos navales, especialmente por su contenido de cromo, níquel y molibdeno. El 140MXC es altamente utilizado para la recuperación y protección de piezas (Marin, 2010), haciendo de esto su ventaja más destacada; su gran fuerza de cohesión se debe al anclaje mecánico obtenido por el aprovechamiento de la energía 
cinética generada por las grandes velocidades alcanzadas durante el proceso (Echeverri y Vargas, 2007).

Los resultados indican que el mejor comportamiento a nivel morfológico se puede lograr a través de la interacción de los parámetros de depósito en función del espesor, microdureza, tamaño de partículas semifundidas y rugosidad (Cooke et al., 2007; Saravanan et al., 2000).

\section{Desarrollo experimental}

Parámetros de diseño usando la técnica Taguchi

En esta investigación se empleó un diseño factorial fraccional $\mathrm{L}_{9}\left(3^{4-2}\right)$, usando un arreglo ortogonal con nueve experimentos. Se usaron cuatro parámetros de proyección como factores del diseño experimental: corriente (I), voltaje $(\mathrm{V})$, presión de aire primaria $(\mathrm{AP})$ y presión de aire secundaria (AS), con tres niveles: 1 (mínimo), 2 (medio) y 3 (máximo), para evaluar el efecto de cada factor. La tabla 1 indica la variación de los parámetros de proyección para los recubrimientos 140MXC-530AS y 140MXC-560AS.

La tabla 2 ilustra el arreglo ortogonal $\mathrm{L}_{9}\left(3^{4-2}\right)$ con los nueve experimentos desarrollados con la técnica Taguchi.
Materiales y equipos

El equipo de proyección térmica es un EUTECTIC 4 del tipo Push/Pull tradicional con rangos operacionales de salida de presión de atomización de 5.5 bar, amperaje de 0 a $350 \mathrm{~A}$ y voltaje de 0 a $50 \mathrm{~V}$ en DC. Los recubrimientos utilizados en el proceso de proyección térmica se seleccionaron por su comportamiento en la recuperación de piezas y resistencia tanto a la corrosión como al desgaste, así como su capacidad de adhesión y cohesión al ser depositados, en forma de monocapas simultáneas, con un espesor calculado para $500 \mu \mathrm{m}$. La composición química de los alambres según los fabricantes (Praxair TAFA; Castolin Eutectic, Arc 530 Wire; Castolin Eutectic Arc 560 Wire) es: 140MXC (Fe, 25Cr, 5B, $6 \mathrm{Mo}, 15 \mathrm{~W}, 3 \mathrm{Mg}, 4 \mathrm{C}, 12 \mathrm{Ni}, 2 \mathrm{Si}), 560 \mathrm{AS}(\mathrm{Fe}, 13 \mathrm{Cr}, 1 \mathrm{Mn}$, $1 \mathrm{Si}, 0.3 \mathrm{C})$ y $530 \mathrm{AS}(\mathrm{Fe}, 0.15 \mathrm{C}, 0.8 \mathrm{Mn}, 0.2 \mathrm{Si}$ ).

El sustrato empleado corresponde a un acero AISI SAE 4340 ampliamente conocido en la industria como uno de los mejores aceros grado maquinaria por su alto contenido de aleación. La composición química obtenida por la técnica de fluorescencia de Rayos X es $(95.2 \mathrm{Fe}$, $1.93 \mathrm{Cr}, 1.64 \mathrm{Ni}, 0.83 \mathrm{Mn}, 0.20 \mathrm{Mo}, 0.13 \mathrm{Cu}, 0.06 \mathrm{~V})$. La preparación superficial del sustrato se realizó por medio del proceso de granallado, con una rugosidad mayor a $50 \mu \mathrm{m}$, según la norma AWS C2.16. Para la eliminación

Tabla 1. Valores de los niveles y factores experimentales utilizados para la proyección térmica por arco eléctrico

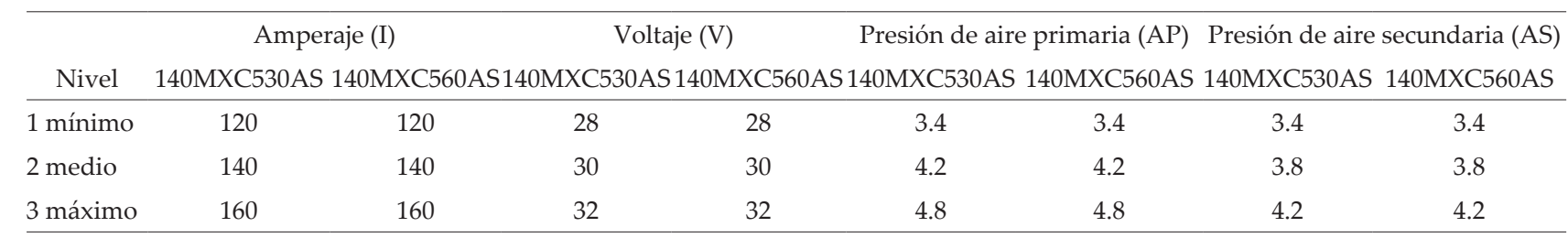

Tabla 2. Diseño experimental arreglo ortogonal $\mathrm{L}_{9}\left(3^{4-2}\right)$

\begin{tabular}{|c|c|c|c|c|c|c|c|c|}
\hline Experimento & Amperaje ( & & Voltaje (V & & Presión de aire & primaria $(\mathrm{AP})$ & Presión de aire & secundaria (AS) \\
\hline Núm. & $140 \mathrm{MXC} 530 \mathrm{AS} 140 \mathrm{~N}$ & MXC560AS1 & $140 \mathrm{MXC530AS} 140 \mathrm{~N}$ & MXC560AS & $140 \mathrm{MXC530AS}$ & 140MXC560AS & 140MXC530AS & 140MXC560AS \\
\hline 1 & 120 & 120 & 28 & 28 & 3.4 & 3.4 & 3.4 & 3.4 \\
\hline 2 & 120 & 120 & 30 & 30 & 4.2 & 3.8 & 4.2 & 3.8 \\
\hline 3 & 120 & 120 & 32 & 32 & 4.8 & 4.2 & 4.8 & 4.2 \\
\hline 4 & 140 & 140 & 28 & 28 & 4.2 & 3.8 & 4.8 & 4.2 \\
\hline 5 & 140 & 140 & 30 & 30 & 4.8 & 4.2 & 3.4 & 3.4 \\
\hline 6 & 140 & 140 & 32 & 32 & 3.4 & 3.4 & 4.2 & 3.8 \\
\hline 7 & 160 & 160 & 28 & 28 & 4.8 & 4.2 & 4.2 & 3.8 \\
\hline 8 & 160 & 160 & 30 & 30 & 3.4 & 3.4 & 4.8 & 4.2 \\
\hline 9 & 160 & 160 & 32 & 32 & 4.2 & 3.8 & 3.4 & 3.4 \\
\hline
\end{tabular}


de las partículas de granalla adheridas a la superficie, se utilizó un limpiador disolvente Magnaflux Spotcheck ${ }^{\circledR}$ SKC-1.

La caracterización de las muestras se realizó por medio de las técnicas de microscopia óptica convencional con un equipo OLYMPUS PME 3, con pulido de las muestras en abrasivos de papel del Núm. 80 a 1500, seguido por un pulido en paño con alúmina de $0.3 \mathrm{y}$ $0.1 \mu \mathrm{m}$; microscopia electrónica de barrido con un equipo JEOL NEOSCOPE 5000 operando con un voltaje de $10 \mathrm{Kv}$ y microscopía de fuerza atómica con un equipo NANOSURF, EASYSCAN2.

\section{Resultados y discusión}

Caracterización del recubrimiento

Composición química por medio de fluorescencia de Rayos X (FRX)

En las figuras 1 y 2 se muestran los espectros del análisis químico por medio de fluorescencia de Rayos $X$ para los recubrimientos 140MXC-530AS y 140MXC-560AS, respectivamente

La tabla 3 contiene los valores en porcentaje de la composición química para las muestras M1 de los recubrimientos 140MXC-530AS y 140MXC-560AS, obtenidas del equipo Fluorescencia de rayos $\mathrm{X}$.
Medición de espesores por medio de microscopía óptica convencional

Las figuras 3 y 4 corresponden a las micrografías a 100X en sección transversal para los experimentos 4 y 3 de los recubrimientos 140MXC-530AS y 140MXC-560AS, respectivamente. Se observa la morfología en forma de monocapa simultánea, identificándose la defectología típica, como porosidades internas, inclusiones de óxidos entre splats $(3 \mathrm{a}, 4 \mathrm{a})$ y grietas entre las capas. Los espesores medidos oscilan entre 158.8 y $478.8 \mu \mathrm{m}$ para el $140 \mathrm{MXC}-530 \mathrm{AS}(3 \mathrm{~b})$ y 130.6 y $415.6 \mu \mathrm{m}$ para el 140MXC-560AS (4b) (Knight y Smith, 1998).

Por el punto de fusión más bajo del nanocompuesto (140MXC) comparado con los otros alambres, este permite una fluidez mayor para llenar con éxito los espacios vacíos (intrasplats), que quedan en el momento de la proyección (Pasandideh et al., 2002).

\section{Ensayo de microdureza}

Las muestras se penetraron con un indentador Vickers sobre la sección transversal del recubrimiento usando una carga de $200 \mathrm{~g}$, ya que mostró menor variación en la prueba preliminar con tiempo de indentación de 30 segundos; de tal manera que la huella generada fuera de tamaño suficiente para no confundirse con alguna porosidad (La barbera et al., 2008). Se realizaron tres in-
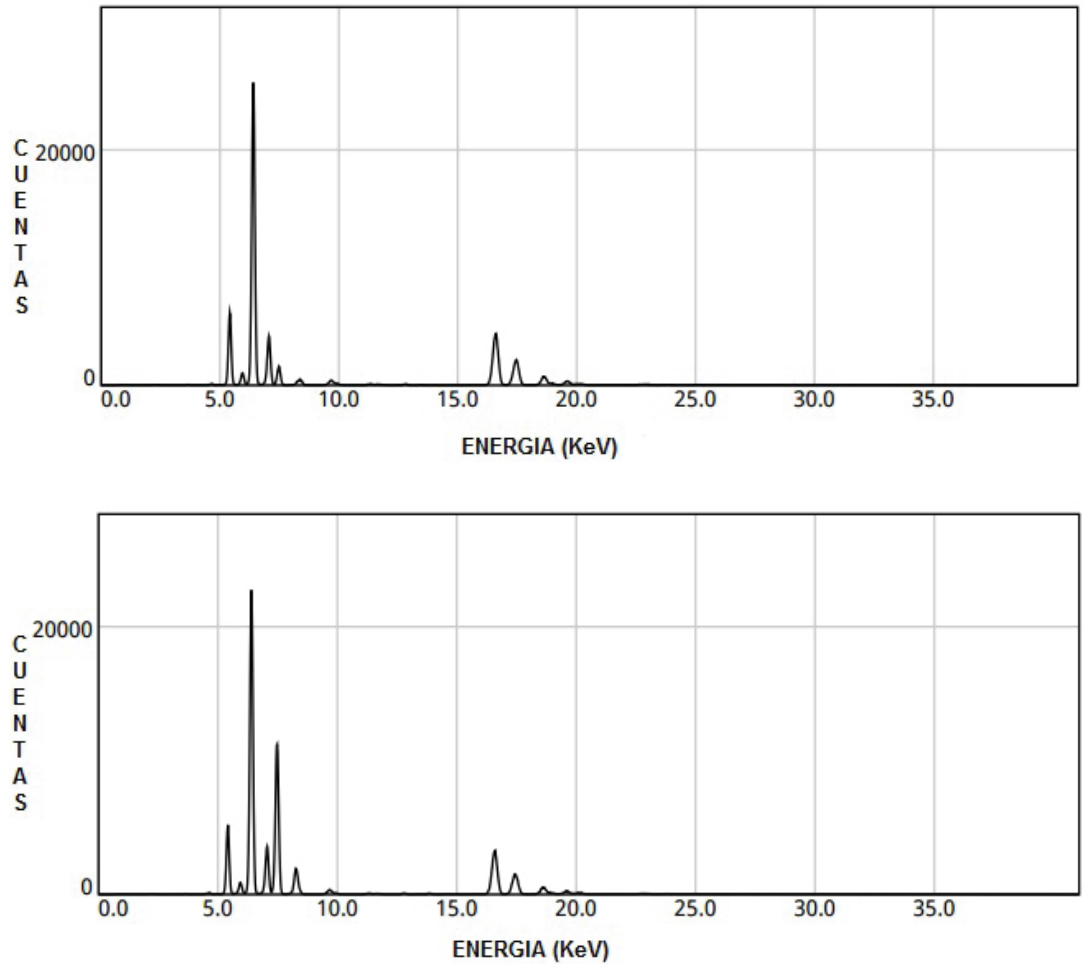

Figura 1. Espectro composición química recubrimiento $140 \mathrm{MXC}-530 \mathrm{AS}$

Figura 2. Espectro composición química recubrimiento 140MXC-560AS 
Tabla 3. Composición química de los recubrimientos

\begin{tabular}{ccccccccc}
\hline Nombre & \multicolumn{10}{c}{ Elemento } \\
\hline M1 140MXC-530AS & $\mathrm{Fe}$ & $\mathrm{Ni}$ & $\mathrm{Cr}$ & $\mathrm{W}$ & $\mathrm{Nb}$ & $\mathrm{Mo}$ & $\mathrm{Ti}$ & $\mathrm{Mn}$ \\
$\%$ & 75.24 & 0.90 & 5.95 & 3.05 & 1.61 & 1.21 & 0.07 & 0.92 \\
M1 140MXC-560AS & $\mathrm{Fe}$ & $\mathrm{Ni}$ & $\mathrm{Cr}$ & $\mathrm{W}$ & $\mathrm{Nb}$ & $\mathrm{Mo}$ & $\mathrm{Ti}$ & $\mathrm{Mn}$ \\
$\%$ & 48.37 & 34.85 & 11.93 & 1.98 & 1.73 & 0.80 & 0.20 & 0.12 \\
\hline
\end{tabular}
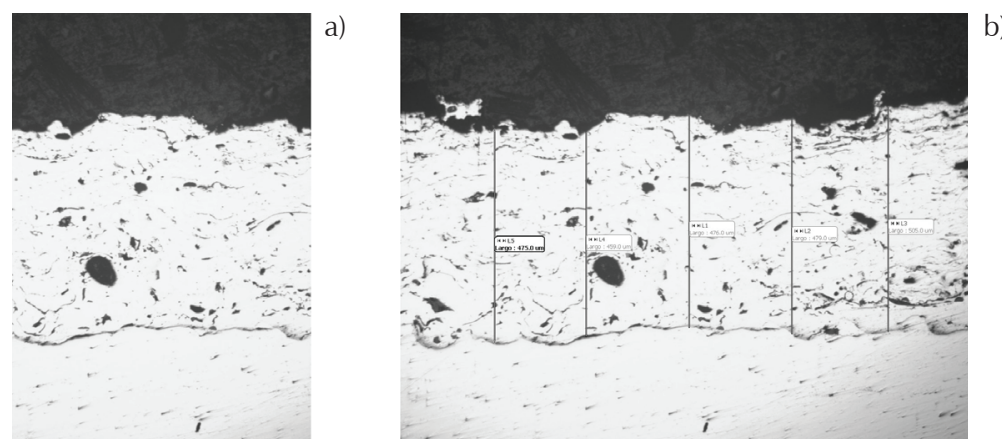

Figura 3. Micrografía del experimento Núm. 4 para el recubrimiento 140MXC-530AS a 100X
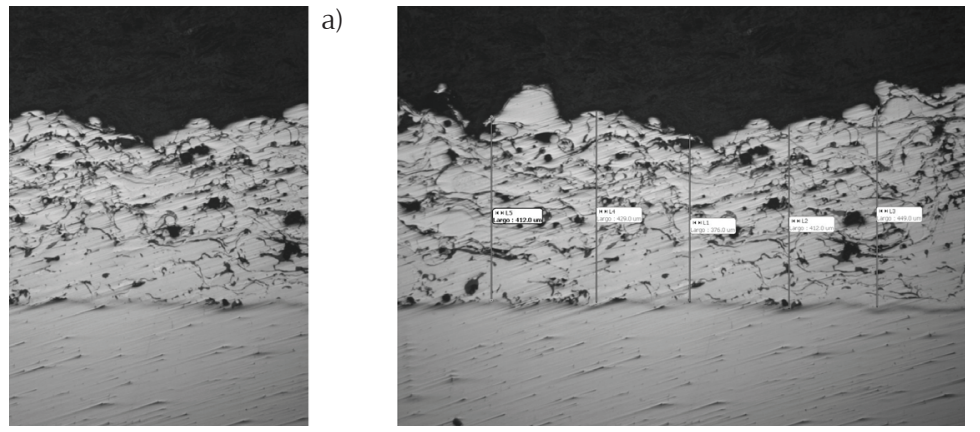

Figura 4. Micrografía del experimento Núm. 3 del recubrimiento 140MXC-560AS a 100X

dentaciones en la zona media y en la interface del depósito con el material base como se muestra en la figura 5.

Identificación morfológica por medio de microscopía electrónica de barrido

Las figuras $6 \mathrm{a}$ y $6 \mathrm{~b}$ corresponden a las micrografías SEM con un acercamiento a $50 \mu \mathrm{m}$, para los experimentos 5 y 6 de los recubrimientos 140MXC-530AS y 140MXC-560AS, respectivamente. Se realizó la toma de micrografías al área superior con el fin de identificar fácilmente la defectología que se presenta en la superficie de los depósitos en función de grietas, inclusiones y poros.
También se observó que la fusión de los materiales en el 140MXC-530AS, generaron una interface entre los splats que desaparecieron, casi por completo, dando lugar a un recubrimiento más compacto, garantizando una mejor protección al sustrato por el crecimiento columnar del depósito, atribuido al buen flujo de calor logrado en la aplicación con la técnica de arco eléctrico (Wang et al., 2001).

Se evidenció tal como lo dice Carvalho y colaboradores (2000) en su investigación, que en el momento en que se expulsan las partículas sufren una descomposición, pero en el momento del impacto contra el sustrato pasan por un proceso de recristalización. De la misma manera que Pasandideh et al. (2002), se encontró que el aumento de voltaje en el proceso crea la producción de 


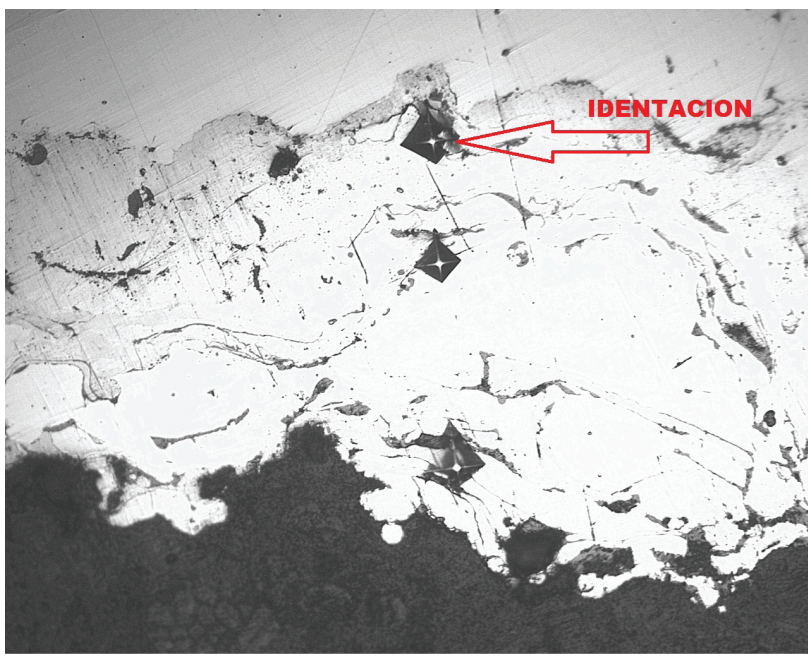

Figura 5. Prueba de microdureza
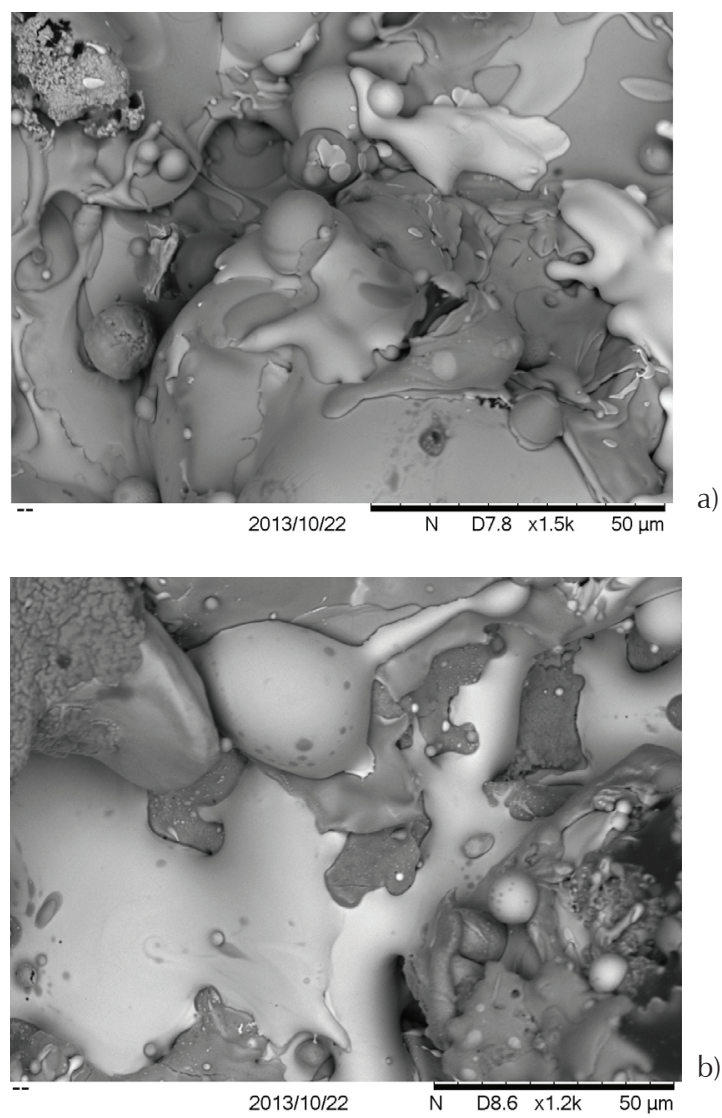

Figura 6. Micrografías SEM, a) experimento Núm. 5 para el recubrimiento 140MXC-530AS, b) experimento Núm. 6 para el recubrimiento 140MXC-560AS

partículas de mayor tamaño, lo que genera un arco inadecuado e inestable entre los alambres que no permite una correcta fusión de los materiales.
Determinación del tamaño de partículas semifundidas

El análisis del tamaño de las partículas semifundidas a la morfología de los recubrimientos obtenidos por la técnica SEM, para el 140MXC-530AS y 140MXC-560AS, se realizó mediante el software "GWYDDION". Se dividieron las imágenes en cuatro sectores como se observa en la figura 7; el software genera un contraste de pixeles a las geometrías seleccionadas y un barrido sobre la superficie de la misma, con el fin de medir el tamaño de partículas semifundidas por medio de una auto-correlación interna de datos estadísticos obtenidos por el programa. La respuesta corresponde a un perfil en dos dimensiones como se muestra en la figura 8.

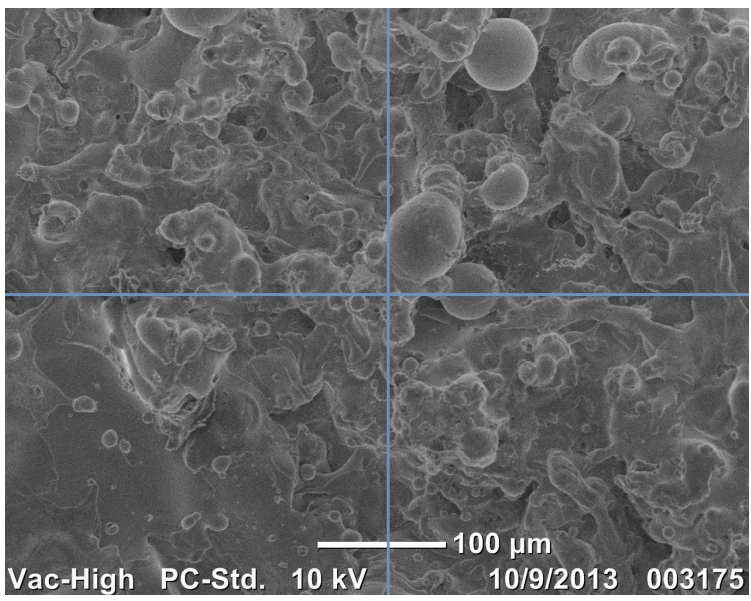

Figura 7. Micrografía SEM dividida en cuatro sectores

Evaluación de la rugosidad por medio de microscopia de fuerza atómica

Las figuras 9 y 10 corresponden al análisis de rugosidad para los experimentos 7 y 8 del 140MXC-530AS y 140MXC-560AS, respectivamente, usando el software "WSxM" del equipo AFM, donde los resultados indican el comportamiento topográfico de las superficies de los recubrimientos, en función de imágenes 3D (9a, 10a), dimensión fractal $(9 b, 10 b)$ y diagrama de rugosidades $(9 c, 10 c)$.

Confirmación de los resultados

Los valores obtenidos para cada experimento de acuerdo con la caracterización de los recubrimientos 140MXC-530AS y 140MXC-560AS, se resumen en la tabla 4. 

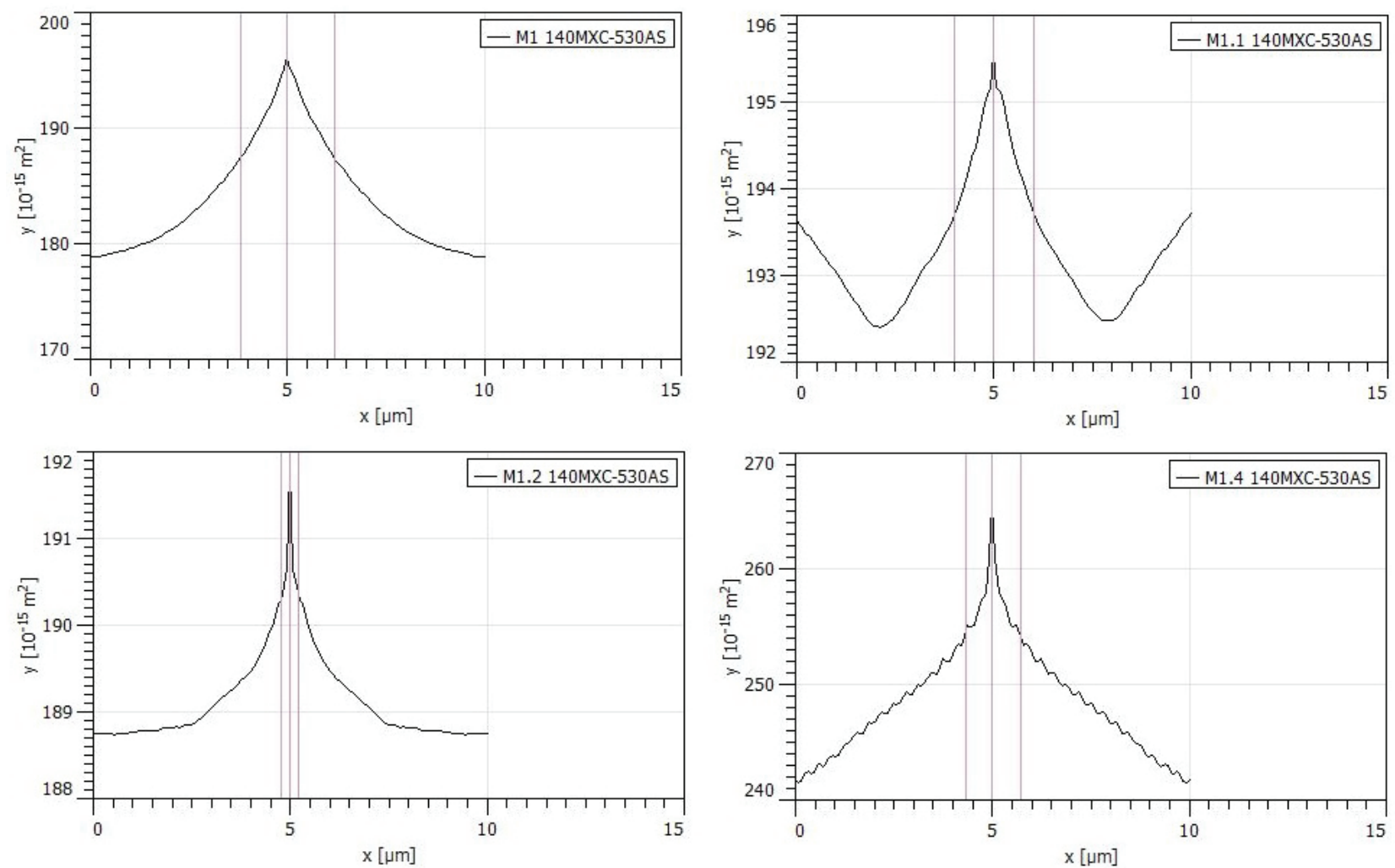

Figura 8. Perfiles de medida de partículas semifundidas
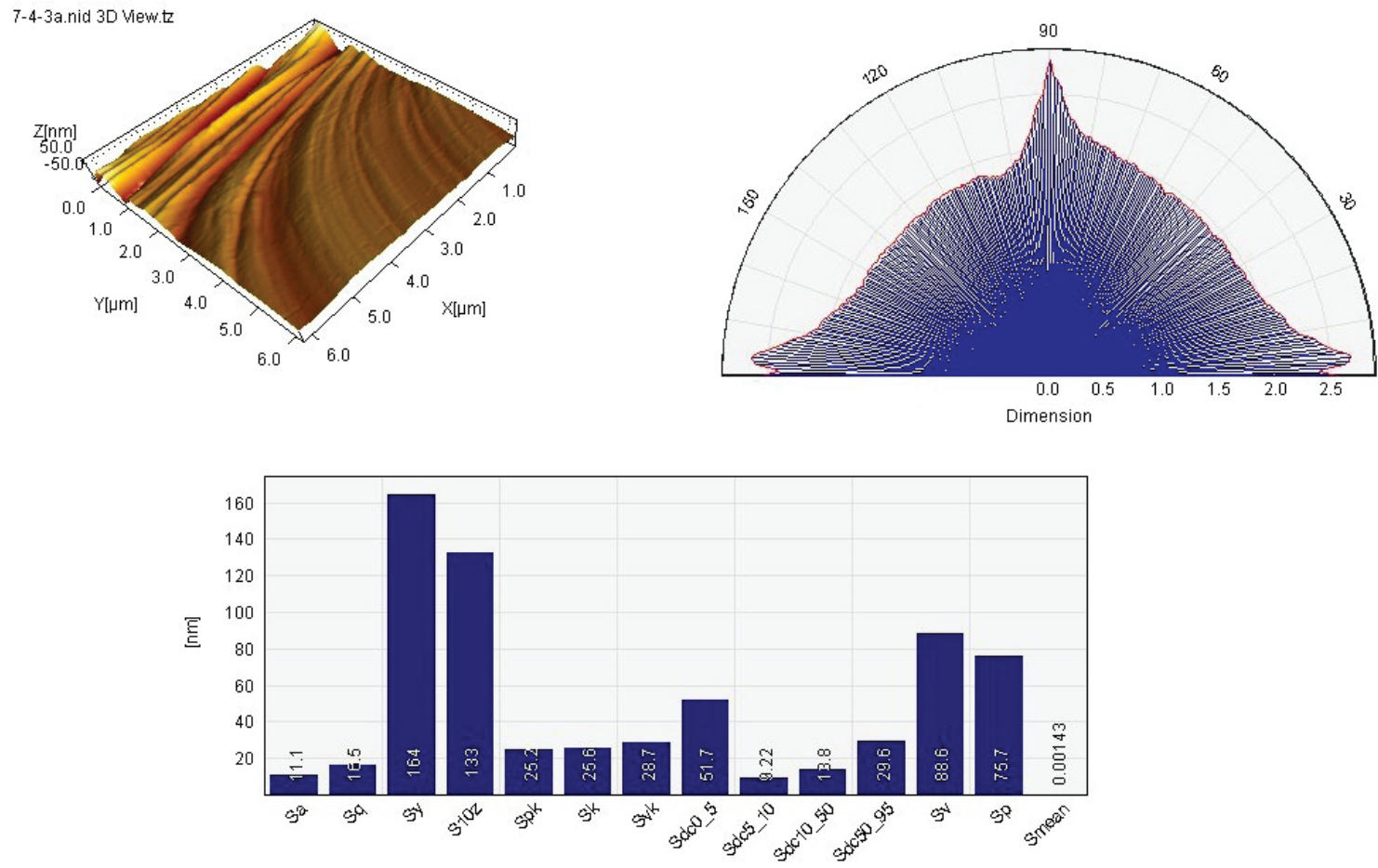

Figura 9. Comportamiento topográfico superficial experimento 7 recubrimiento 140MXC-530AS 

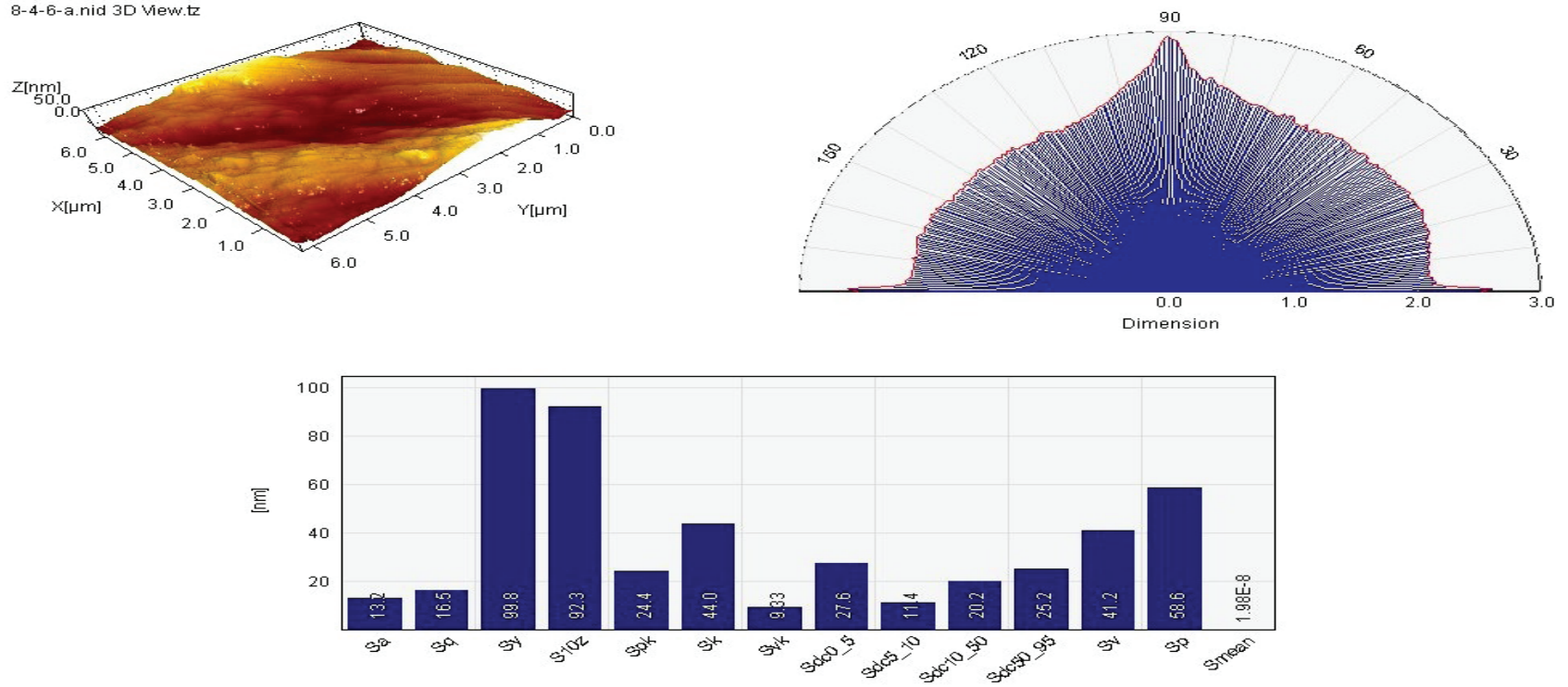

Figura 10. Comportamiento topográfico superficial experimento 8 recubrimiento 140MXC-560AS

Tabla 4. Valores obtenidos en la caracterización de los recubrimientos

\begin{tabular}{|c|c|c|c|c|c|c|c|c|}
\hline \multirow{2}{*}{$\begin{array}{l}\text { Experimento } \\
\text { Núm. }\end{array}$} & \multicolumn{2}{|c|}{ Espesores $(\mu \mathrm{m})$} & \multicolumn{2}{|c|}{ Microdureza (Hv) } & \multicolumn{2}{|c|}{ Tamaño partículas $(\mu \mathrm{m})$} & \multicolumn{2}{|c|}{ Rugosidad (nm) } \\
\hline & 140MXC530AS & 140MXC560AS & 140MXC530AS & 140MXC560AS & 140MXC530AS & 140MXC560AS & 140MXC530AS & 140MXC560AS \\
\hline 1 & 252.2 & 244.8 & 584.53 & 186.51 & 1.55 & 0.87 & 376 & 163 \\
\hline 2 & 254.8 & 216.2 & 148.93 & $218, .30$ & 1.39 & 1.19 & 251 & 431 \\
\hline 3 & 158.8 & 415.6 & 164.86 & 422.86 & 0.85 & 1.33 & 218 & 155 \\
\hline 4 & 478.8 & 215.2 & 189.66 & 487.73 & 1.11 & 0.89 & 510 & 662 \\
\hline 5 & 368.4 & 228.0 & 294.76 & 121.62 & 0.79 & 1.37 & 282 & 223 \\
\hline 6 & 306.6 & 264.2 & 520.93 & 314.98 & 1.37 & 0.74 & 338 & 467 \\
\hline 7 & 404.2 & 361.8 & 351.60 & 324.60 & 2.76 & 0.89 & 164 & 162 \\
\hline 8 & 418.0 & 130.6 & 154.37 & 298.73 & 1.20 & 1.76 & 268 & 99.8 \\
\hline 9 & 361.2 & 183.4 & 173.30 & 202.13 & 1.34 & 1.01 & 263 & 140 \\
\hline
\end{tabular}

Con estos resultados se calculó el promedio para cada uno de los niveles experimentales en función de los parámetros de depósito correlacionados a las características de los recubrimientos producidos, representados en las figuras 11 a 18.

Las figuras 11 y 12 muestran el comportamiento de los recubrimientos en función del espesor. En el recubrimiento 140MXC-530AS el mejor resultado se presenta en el conjunto de parámetros de depósito I3, V1, AP2 y AS3, mientras tanto, para el recubrimiento 140MXC560AS estuvo en I1, V3, AP3 y AS2.

Las figuras 13 y 14 muestran el comportamiento de los recubrimientos en función de la microdureza. En el recubrimiento $140 \mathrm{MXC}-530 \mathrm{AS}$ el mejor resultado se presenta el en conjunto de parámetros de depósito I2,
V1, AP1 y AS1, mientras que para el recubrimiento 140MXC-560AS, estuvo en I2, V1, AP2 y AS3.

Las figuras 15 y 16 muestran el comportamiento de los recubrimientos en función del tamaño de partículas semifundidas. En el recubrimiento 140MXC-530AS el mejor resultado se presenta en el conjunto de parámetros de depósito I2, V2, AP2 y AS3, mientras que para el recubrimiento 140MXC-560AS, estuvo en I2, V1, AP2 y AS2.

Las figuras 17 y 18 muestran el comportamiento de los recubrimientos en función de la rugosidad. En el recubrimiento 140MXC-530AS el mejor resultado se presenta en el conjunto de parámetros de depósito I3, V2, AP3 y AS2, mientras que para el recubrimiento 140MXC-560AS, estuvo en I3, V2, AP3 y AS1. 


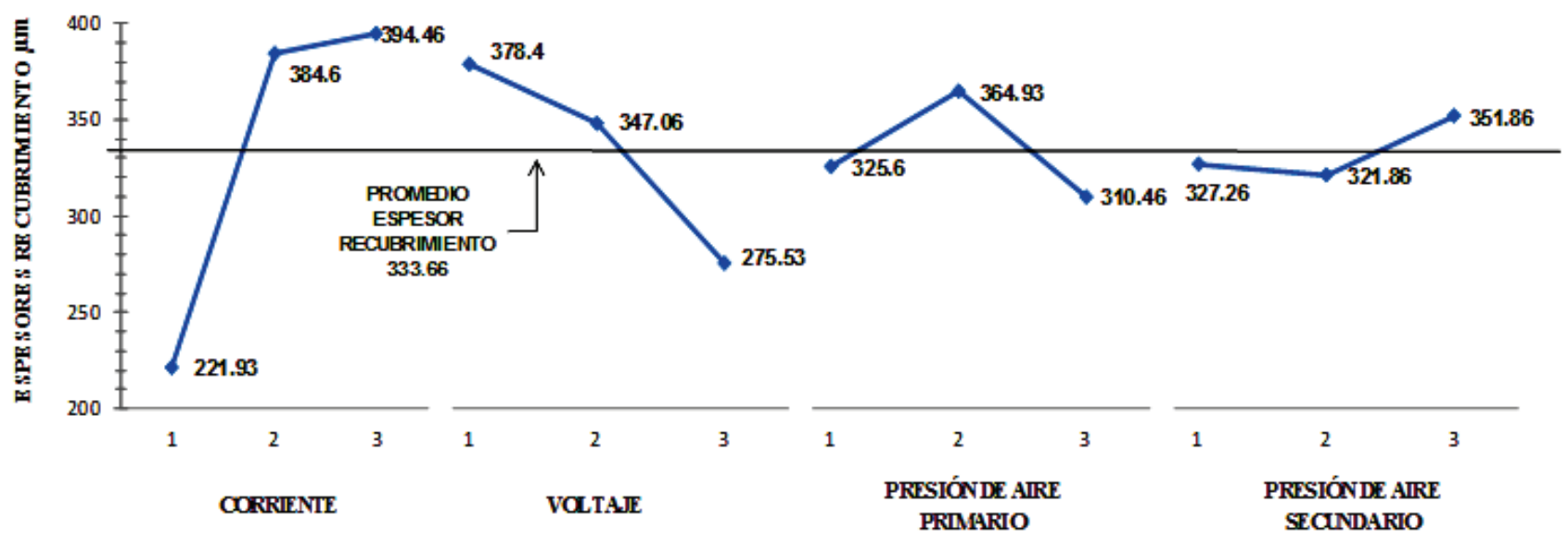

Figura 11. Parámetros de depósito vs espesor para el recubrimiento140MXC-530AS

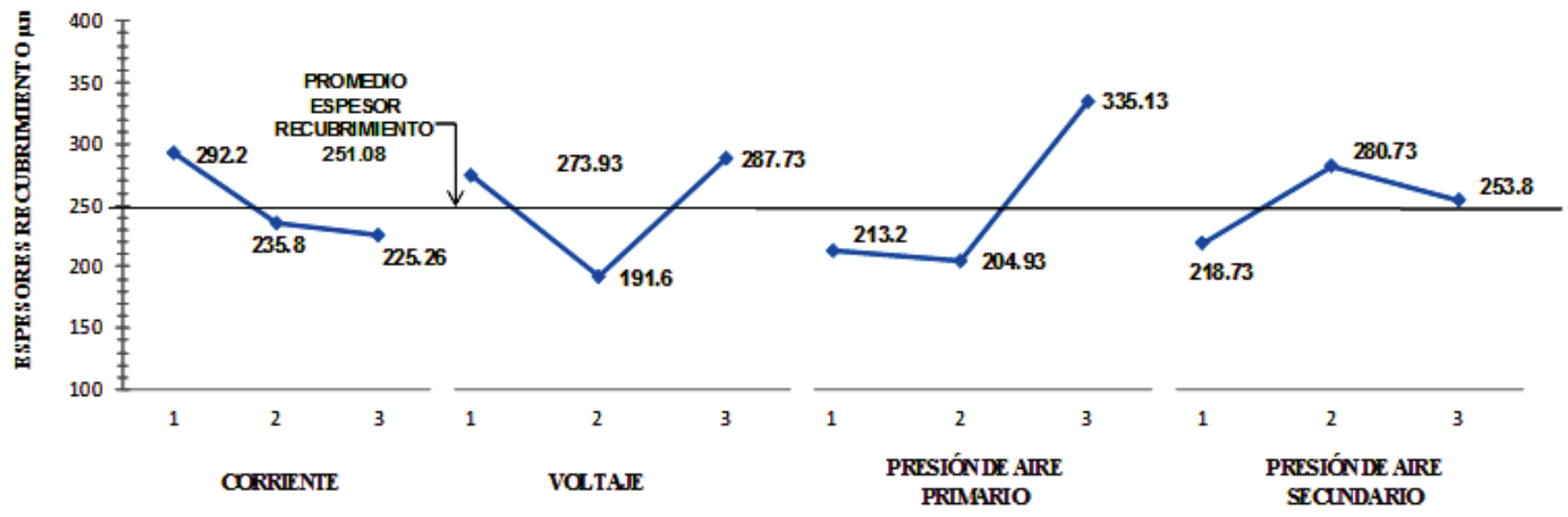

Figura 12. Parámetros de depósito vs espesor para el recubrimiento140MXC-560AS

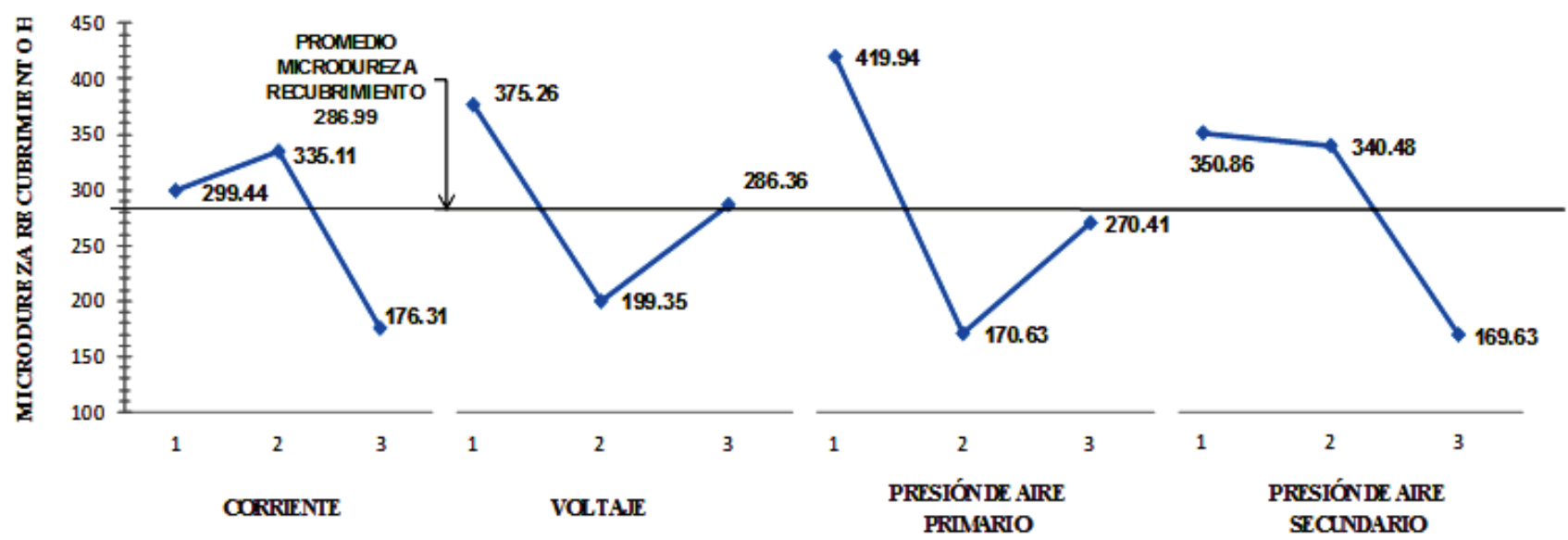

Figura 13. Parámetros de depósito vs microdureza para el recubrimiento 140MXC-530AS 


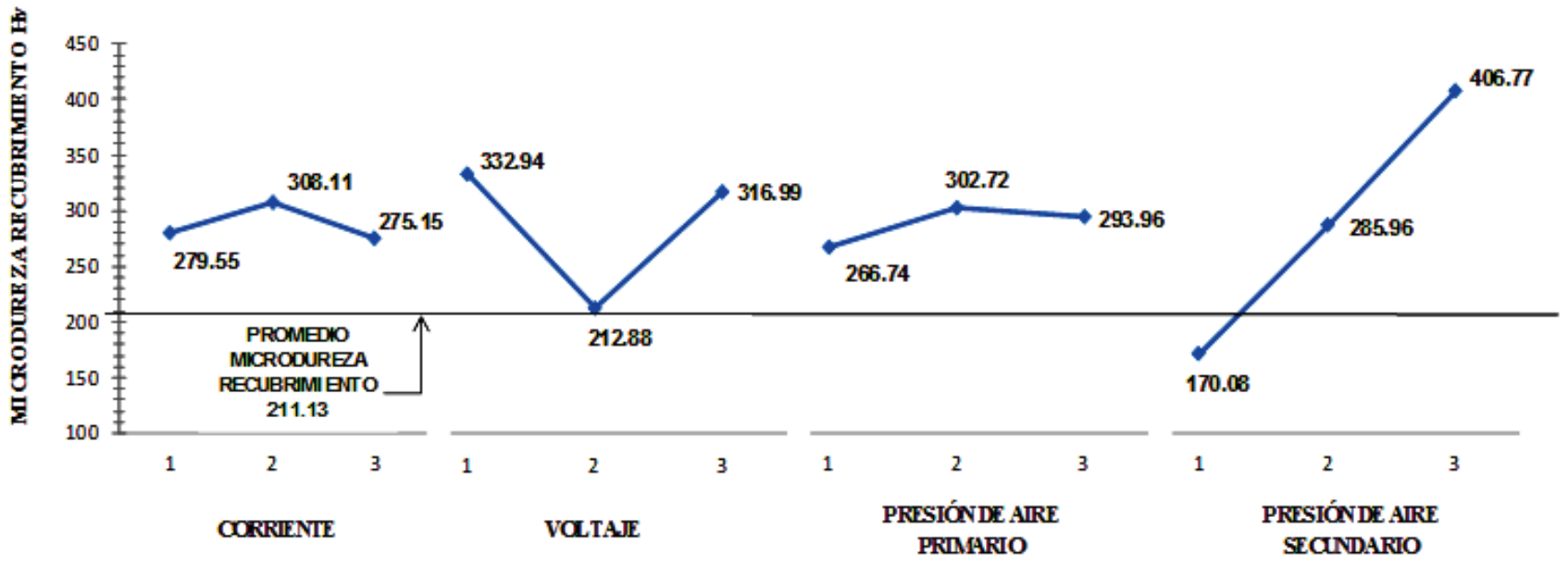

Figura 14. Parámetros de depósito vs microdureza para el recubrimiento 140MXC-560AS

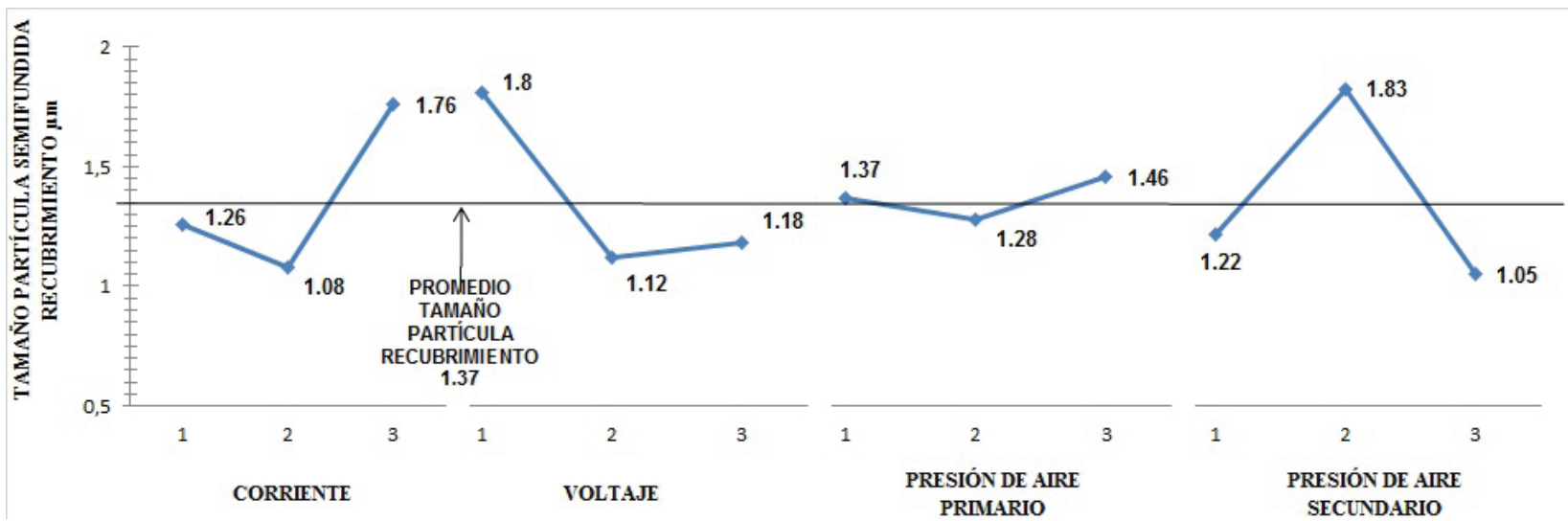

Figura 15. Parámetros de depósito vs tamaño de partículas semifundidas para el recubrimiento 140MXC-530AS

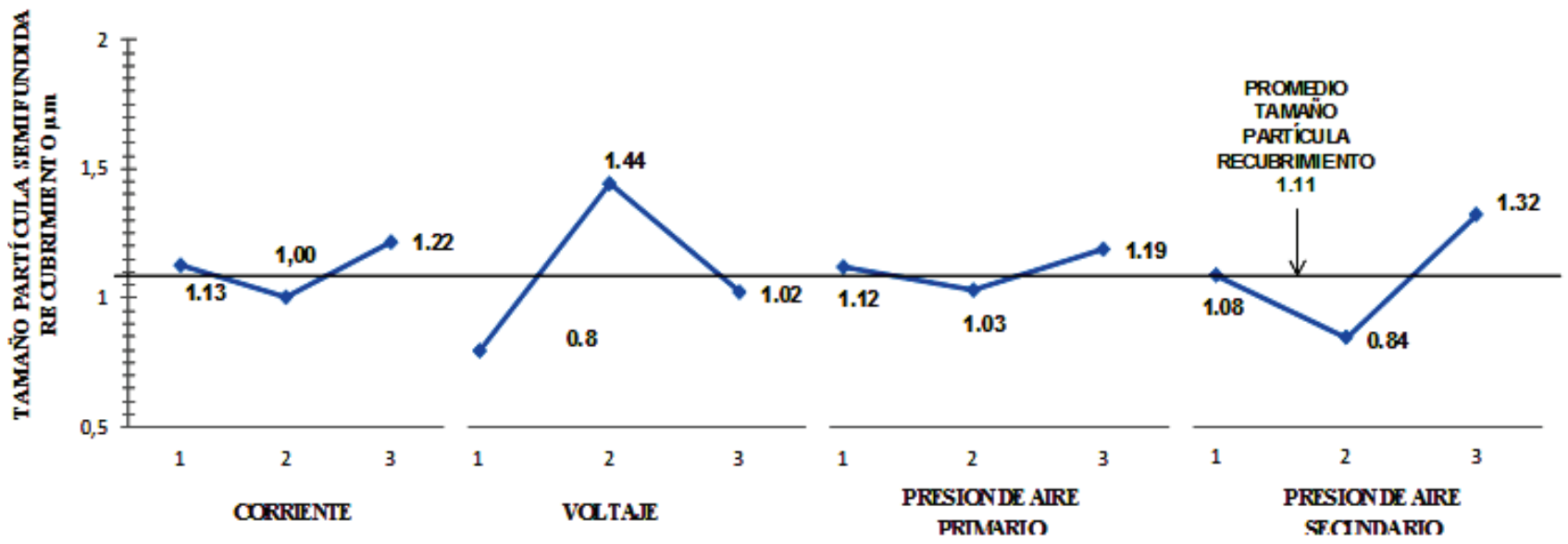

Figura 16. Parámetros de depósito vs tamaño de partículas semifundidas para el recubrimiento 140MXC-560AS 


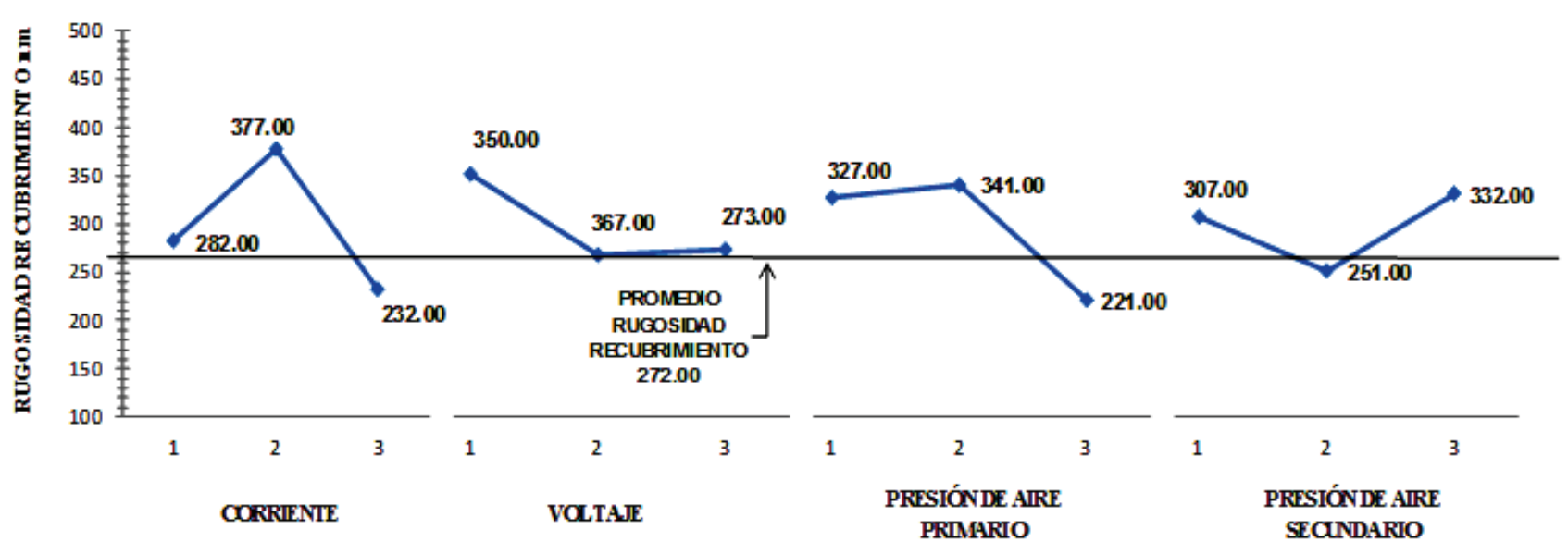

Figura 17. Parámetros de depósito vs rugosidad para el recubrimiento 140MXC-530AS

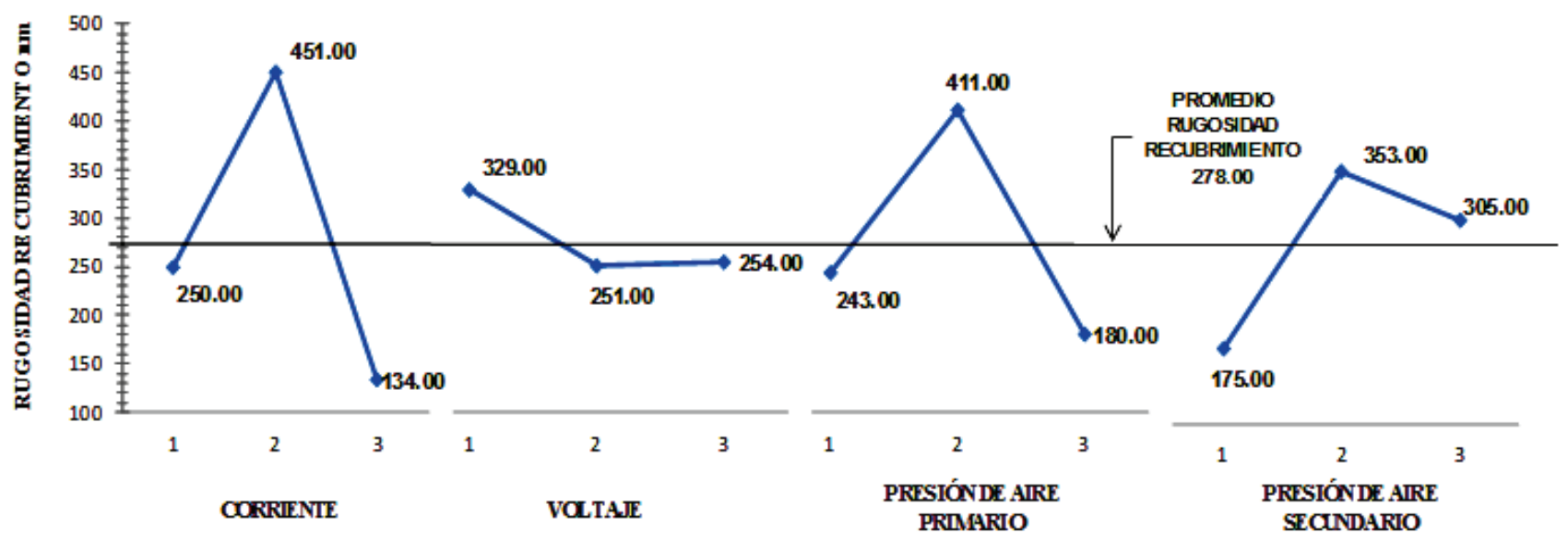

Figura 18. Parámetros de depósito vs rugosidad para el recubrimiento 140MXC-560AS

\section{Conclusiones}

Los recubrimientos 140MXC-530AS Y 140MXC-560AS se obtuvieron mediante la técnica de aspersión térmica por arco eléctrico, utilizando un equipo Eutectic System4. Se siguió el método experimental Taguchi, cuyos parámetros de proyección fueron corriente, voltaje, presión de aire primario y presión de aire secundario.

Para el espesor de los recubrimientos, la mejor condición del 140MXC-530AS corresponde al parámetro I3 (corriente 160A), con un valor de $394.46 \mu \mathrm{m}$, mientras que para el 140MXC-560AS se atribuye al parámetro AP3 (presión de aire primario $4.8 \mathrm{Bar}$ ), el mejor comportamiento con un valor de $335.13 \mu \mathrm{m}$.

La microdureza presentó su mejor comportamiento en función del AP1 (aire primario 3.4 Bar) con un valor de 419.94 Hv para el 140MXC-530AS y AS3 (presión de aire secundario 4.2 Bar) con un valor de $406.77 \mathrm{Hv}$ para el 140MXC-560AS.

Según el tamaño de partículas semifundidas el valor de $1.05 \mu \mathrm{m}$, es el mejor atributo para el 140MXC-530AS en función de AS3 (presión de aire secundario 4.8 Bar) $\mathrm{y}$ de $0.80 \mu \mathrm{m}$ en el 140MXC-560AS en función de V1 (voltaje de 28V).

En cuanto a la rugosidad para el 140MXC-530AS, el valor más bajo presentado corresponde a $221 \mu \mathrm{m}$ en función de AP3 (presión de aire primario 4.8 Bar) y para 140MXC-560AS el valor fue de $134 \mu \mathrm{m}$ en función de I3 (corriente $160 \mathrm{~A}$ ).

En esta fase experimental el método Taguchi permitió establecer que existen factores experimentales comunes dentro del mejor comportamiento de los recubri- 
mientos producidos cuyas alternativas serían para el 140MXC-530AS I2, V1, AP2, AS3 o I3, V2, AP2, AS3; para el 140MXC-560AS I2, V1, AP3, AS2 o I2, V1, AP3, AS2.

\section{Referencias}

Carvalho-De Cecilia et al. Caracterización de recubrimientos de hidroxiapatita depositadas sobre la aleación Ti6Al7Nb a través de aspersión térmica a plasma, 2000, pp. 49-53.

Castolin Eutectic. Data Sheet. Euctronic Arc 530 Wire.

Castolin Eutectic. Data Sheet. Euctronic Arc 560 Wire.

Cooke K. et al. Optimization of the electric wire arc-spraying process for improved wear resistance of sugar mill roller shells. Surface \& Coatings Technology, volumen 202, 2007:185-188.

Echeverri J.B.S., Vargas F. Estudio de la resistencia al desgaste de recubrimientos depositados mediante proyección térmica sobre acero al carbono. Scientia et Technica, volumen XIII, 2007.

Handbook of Thermal Spray Technology, ASM International, 2004, p. 3.

Kelly R. Corrosion technology, Electrochemical thermodynamics and kinetics of relevance to corrosion, Marcel Dekker, 2003.

Knight R. y Smith R.W. Thermal spray forming of materials. Powder Metal Technologies and Applications, volumen 7, 1998: 11.

Knight R. Thermal spray forming of materials, powder metal technologies and applications. ASM Handbook, ASM International, volumen 7, 1998, pp. 408-419.

La Barbera-Sosa J.G. et al., Microstructural and mechanical characterization of Ni-base thermal spray coatings deposited by HVOF. Surface and Coatings Technology, volumen 202 (número 18): 2008: 4552-4559.

Pasandideh-Fard M. et al. Splat shapes in a thermal spray coating process: simulations and experiments. Journal of Thermal Spray Technology, volumen 11 (número 2) 2002.
Praxair TAFA Technical. Data Bulletin. File 1.9.2., 140 MXC., Nano Composite Wire.

Quaranta N. et al. Análisis comparativo de recubrimientos sobre aluminio, CONAMET/SAM, 2004.

Salvador M. Recubrimientos: protección contra corrosión y el desgaste, Instituto de Tecnología de Materiales, 2011.

Saravanan P., Selvarajan V., Rao D.S., Joshi S.V., Sundararajan G., Mater J. Manuf. Process., volumen 10, 2000: 459-472.

Thermal Spray Society Training Committee. Handbook of Thermal Spray Technology, 1a ed., Davis J.R., Davis \& Associates, editors, United States of America, ASM International, 2004, 338 p.

Villar-Camilo M Revista Metal Actual, 2010: 45.

Wang G.X., Prasad V., Sampath S. Rapid solidification in thermal spray deposition: microstructure and modeling. Sadhana, volumen 26 (número 1): 2001: 35-57.

\section{Este artículo se cita:}

\section{Citación estilo Chicago}

Rojas-Molano Héctor Fernando, Jhon Jairo Olaya-Flórez, Cesar Andrés Molina-González. Caracterización morfológica de los recubrimientos 140MCX-530AS y 140MCX-560AS usando la técnica de proyección térmica por arco eléctrico. Ingeniería Investigación y Tecnología, XVII, 01 (2016): 1-13.

\section{Citación estilo ISO 690}

Olaya-Flórez J.J., Rojas-Molano H.F., Molina-González C.A. Caracterización morfológica de los recubrimientos 140MCX-530AS y 140MCX-560AS usando la técnica de proyección térmica por arco eléctrico. Ingeniería Investigación y Tecnología, volumen XVII (número 1), enero-marzo 2016: 1-13. 


\section{Semblanzas de los autores}

Héctor Fernando Rojas-Molano. Es ingeniero metalurgico y de materiales por la Universidad Libre de Colombia, es candidato a doctor en ingeniería, ciencia y tecnología de materiales de la Universidad Nacional de Colombia. Docente investigador de la Facultad de Ingeniería y director del Departamento de Ciencias Básicas de la Universidad Libre en las areas de corrosión, soldadura e inspeccion y ensayos. Director de proyectos de investigación en convenio con el SENA, COTECMAR, UNAL y Ortomac. Asesor y consultor para empresas de la industria metalúrgica, metalmecánica, fundición, aeronáutica, automotriz y naval. Cuenta con publicaciones en la revista ShipScience and Technologyy la revista AVANCES Investigación en Ingeniería.

Jhon Jairo Olaya-Florez. Es ingeniero metalurgico de la Universidad Libre de Colombia, magister en materiales y procesos de manufactura por la Universidad Nacional de Colombia y doctor en ingeniería por la Universidad Nacional Autónoma de México. Es profesor asociado de la Facultad de Ingeniería y produde investigación en el área de nanomateriales, caracterización y procesamiento de materiales por plasma.

Cesar Andres Molina-González. Estudiante del programa de ingeniería mecánica de la Universidad Libre de Colombia. Investigador auxiliar del proyecto de caracterización por microscopia óptica convencional, electrónica de barrido y de fuerza atómica para recubrimientos obtenidos por la técnica de proyección térmica por arco eléctrico. Cuenta con experiencia técnica en el manejo de equipos de control numérico en el procesamiento de materiales por arranque de viruta. 